American Journal of Pharmacology and Toxicology 7 (1): 19-26, 2012

ISSN 1557-4962

(C) 2012 Science Publications

\title{
Hepatic and Renal Histological Alterations Induced by Topical Hydroquinone Administration
}

\author{
${ }^{1}$ Bashir M. Jarrar, ${ }^{2}$ Maher M. Alenezi, ${ }^{1}$ Abdullah Al-Hiri and ${ }^{3}$ Yazun Jarrar \\ ${ }^{1}$ Department of Clinical Laboratory Sciences, \\ College of Applied Medical Sciences, Al-Jouf University, Saudi Arabia \\ ${ }^{2}$ School of Medicine, Al-Jouf University, Saudi Arabia \\ ${ }^{3}$ Department of Pharmacology, School of Medicine, Inje University, South Korea
}

\begin{abstract}
Problem statement: Although the regulatory agencies of the United States and Europe banned hydroquinone in cosmetics skin lightening but it is still used in most of Middle East countries, including Saudi Arabia. Few studies were carried out on the histological alterations of hydroquinone on kidney and liver. Approach: The present study was carried out to investigate the histological alterations in liver and kidney caused by hydroquinone topical administration. Results: The induced histological alterations in the liver were mainly hydropic degeneration, bile duct hyperplasia and glycogen depletion. The kidney of treated rabbits showed hydropic degeneration, hyaline casts, congestion, perivascular edema and fibrosis together with lymphocytic aggregation. The skin showed hyperkeratosis, lymphocytic and eosinophilic infiltration together with congestion of dermal blood vessels. Conclusion: Taken together, the histological findings of this study indicate that chronic exposure to hydroquinone produce significant histological alterations that might affect the liver and kidney.
\end{abstract}

Key words: Hydroquinone, eldoquin, histological alterations, topical administration, hepatic adenoma

\section{INTRODUCTION}

Hydroquinone has been used for decades in treatment of dyschromia specially hyperpigmentation such as melasma (Zawar and Mhaskar, 2004; Makropoulos and Alexopoulos, 2006). Also, it functions in cosmetics as a antioxidant or polymerization agent in a number of topical skin creams (Boyle and Kennedy, 1986; Draelos, 2007; Costa et al., 2010; Anderson et al., 2010).

Studies have been reported hydroquinone to produce renal tubule hyperplasia, adenomas and chronic progressive nephropathy by enhancing renal cell proliferation (Boyle and Kennedy, 1985; Hard et al, 1997; Karamagi et al., 2001). Other reports indicated that hydroquinone to increase the incidence of leukemia and to inhibit apoptosis of neoplastic cells in experimental animals while genotoxicity and mutagenicity studies on this drug were almost negative (Hazel et al., 1996; McGregor, 2007; Anderson et al., 2010). Also, it has the potential of exogenous ochronosis and affects the host defense in infectious diseases by lowering the level of neutrophils (Westerhof and Kooyers, 2005; Ribeiro et al., 2011).
Studies revealed that the potential hazards of topical application of hydroquinone is more than oral exposure (Kooyers and Westerhof, 2006). However high doses of oral administration of hydroquinone showed that it affects the feed consumption and/or body weight and the function of the central nervous system (Devillers et al., 1990; Murphy et al., 1992; DeCaprio, 1999; Joseph et al., 1998).

The usage of hydroquinone in cosmetics skin lightening in Europe has been banned since 2001 due to its effects (Kooyers and Westerhof, 2006; Makropoulos and Alexopoulos, 2006). Also regulatory agencies of the United States banned hydroquinone on the base of reports confirmed the induction of exogenous ochronosis in human, hepatic adenoma and renal adenoma in experimental animals over extended time period (Draelos, 2007; Levitt, 2007). However, it is still used in most of Middle East countries, including Saudi Arabia and is available in the pharmacy as topical formula, in concentrations of 2 and $4 \%(\mathrm{~W} / \mathrm{W})$.

There are a few studies about the histological alterations of hydroquinone on kidney and liver (Boatman, 1996; Topping et al., 2007). Also, most of these studies used oral or intra-peritoneal routes of

Corresponding Author: Bashir M. Jarrar, Department of Clinical Laboratory Sciences, College of Applied Medical Sciences, AL-Jouf University, P.O. Box (2014), Skaka-Al-Jouf, Saudi Arabia Tel: 00966-543647100 Fax: 00966-46257328 
administration which are not clinically administrated (Boatman, 1996; McGregor, 2007). So, the present study was carried out to investigate the histological changes in liver and kidney caused by topical administration of hydroquinone.

\section{MATERIALS AND METHODS}

Experimental animals: A total of 24 adult female rabbits (weight 400-1500 gm) were kept in animal house cages where each contained one animal and divided into four groups (6 animals each). The temperature and the relative humidity were monitored daily during the period of experimental course where the temperature in the experimental laboratory was $25 \pm 2.06^{\circ} \mathrm{C}$ and relative humidity of $47.44 \pm 9.29$. Further, animals were maintained under natural lighting of $12 \mathrm{~h}$ daylight and $12 \mathrm{~h}$ darkness.

The animals were subjected to eldoquin (hydroquinone $2 \% \mathrm{w} / \mathrm{w}$ ) topical administration for 6 weeks as follows:

Group I: Control group: the members of this group were not subjected to hyroquinone administration.

Group II: Hyroquinone $(2 \%, w / w)$ cream was applied on the total outer surface of the right ear of each member.

Group III: Hyroquinone $(2 \%, w / w)$ cream was applied on the total outer surface of both ears of each member.

Group IV: Hyroquinone $(2 \%, \mathrm{w} / \mathrm{w})$ cream was applied on the abdomen $\left(2 \mathrm{gm} / \mathrm{cm}^{2}\right)$ after hair shaving of each member.

Animals were euthanized and fresh biopsies were taken from the kidney, liver, abdominal skin and both ears of each rabbit. Tissue specimens were fixed in $10 \%$ neutral buffer formalin and then dehydrated in ascending grades of ethanol (70, 80, 90 and 100\%). Dehydration was followed by clearing the tissue samples in 2 changes of xylene before being impregnated with 2 changes of melted paraffin wax, embedded and blocked out. Tissue sections (4-5 $\mu \mathrm{m})$ were stained with hematoxylin eosin, Mallory trichrome and Periodic Acid-Schiff (PAS) according to the methods described by Taib et al. (2004); Pearse (1985); Carleton et al. (1980) and Bancroft and Stevens (1990) for the conventional histological procedures.

\section{RESULTS AND DISCUSSION}

Exposure to $2 \%(\mathrm{w} / \mathrm{w})$ hydroquinone cream has produced the following histological alterations in the liver of treated rabbits.
Diffuses and hydropic degeneration: The hepatocytes of hydroquinone treated rabbits were swollen and showed clear cytoplasm. This alteration was seen in all hydroquinone treated rabbits (Fig. 1a).

Bile duct hyperplasia: Marked bile duct hyperplasia was seen in the treated rabbits specially those subjected to hydroquinone on both ear auricle (Fig. 1b and c).

Glycogen depletion: Periodic Acid-Schiff (PAS) stained showed evident partial glycogen depletion in the hepatocytes of treated rabbits in comparison with the control liver. Hydroquinone exposure had has produced significant reduction in the liver glycogen in a heterogeneous pattern. The reduction was mainly in the hepatocytes adjacent to the peripheral zone while the pericentral and the midzone hepatocytes were less affected (Fig. 1d).

Also exposure to $2 \%(\mathrm{w} / \mathrm{w})$ hydroquinone has produced the following histological alterations in the kidney.

Hydropic degeneration: Renal tubules showed hydropic degeneration with deteriorated cytoplasm (Fig. 2a). This alteration was more prominent in rabbits exposed to hydroquinone in both ears.

Hyaline casts: The lumen of some renal tubules showed hyaline casts (Fig. 2b).

Congestion: The kidney of treated rabbits showed dilatation and congestion of intertubular blood capillaries in comparison with the control ones (Fig. 2c). Rabbits of group 4 were more affected than other treated groups.

Perivascular edema: The kidney of hydroquinone treated rabbits showed occasional perivascular edema (Fig. 2d). Connective tissues surrounding renal blood vessels became widely separated from the adventia of these vessels due to accumulation of the edematous fluid. This change was more prominent in rabbits exposed to hydroquinone in both ears in comparison to the animals of other treated groups.

Perivascular fibrosis: Interstitial perivascular fibrosis was noted in renal tissue of the hydroquinone treated rabbits (Fig. 2e). This change was seen in the kidney of all treated rabbits.

Perivascular lymphocytic aggregation: An occasionally aggregation of mononuclear inflammatory cells mainly lymphocytes were seen next to the renal veins (Fig. 2f). 


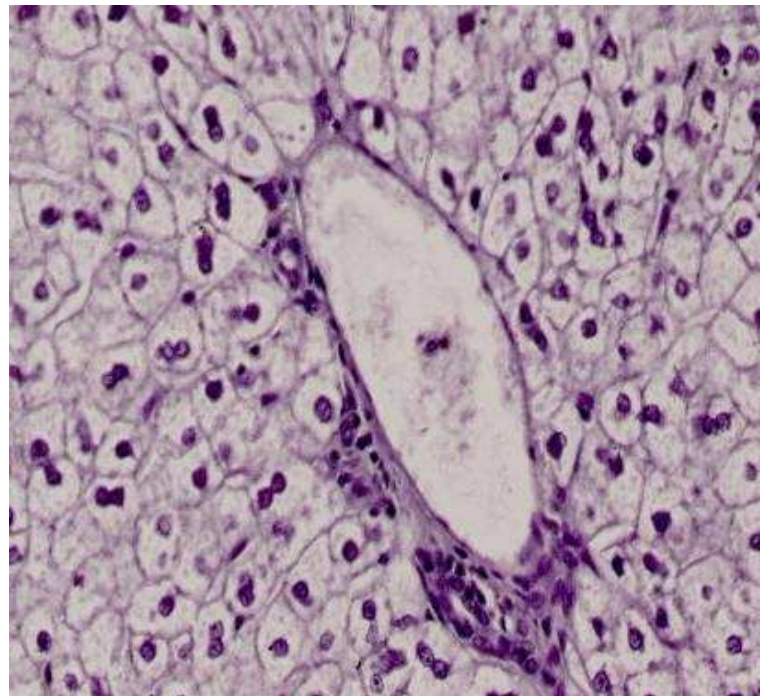

(a)

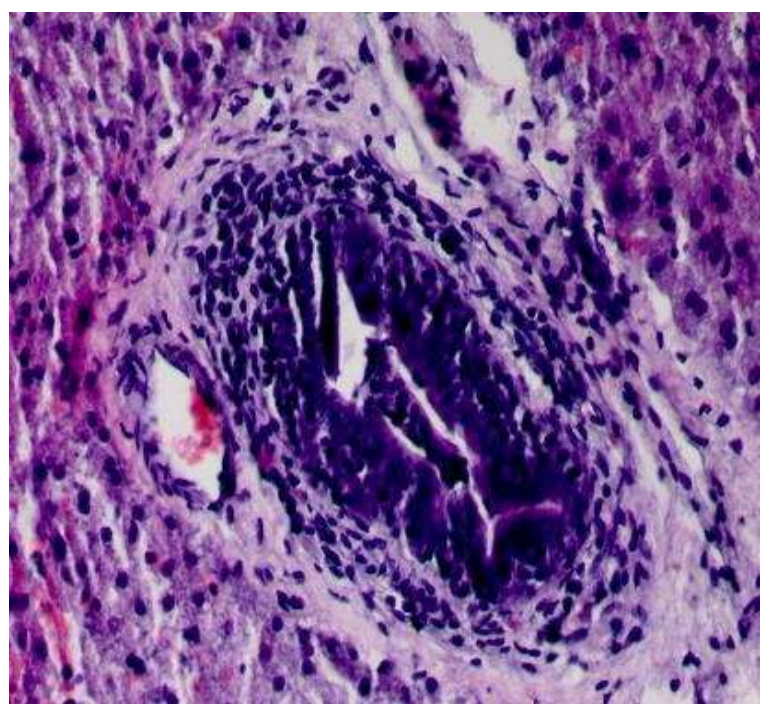

(c)

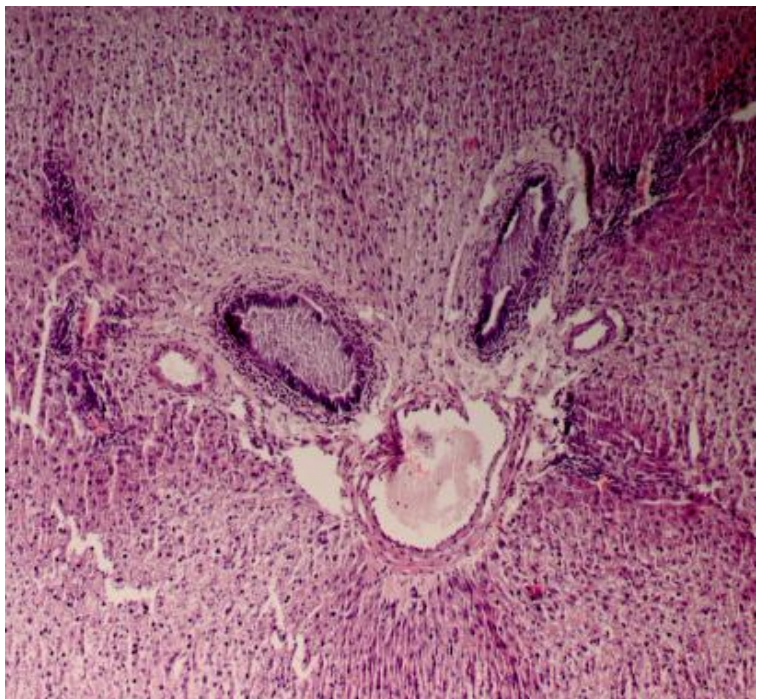

(b)

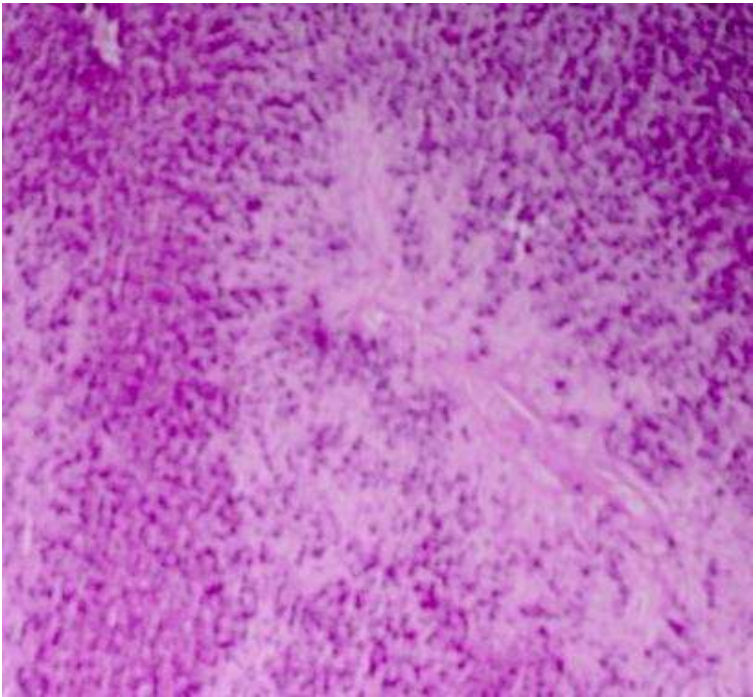

(d)

Fig. 1: Light micrograph section in the liver of rabbit exposed to eldoquin (hydroquinone, 2\%, w/w) showing (a) Diffuses and hydropic degeneration. H\&E stain; (b) Bile duct hyperplasia. H\&E stain; (c) Thickening bile duct epithelium. Trichrome stain; (d) Glycogen depletion mainly in the periportal space. PAS stain

The following histological alterations in the skin subjected to the drug were also seen.

Hyperkeratosis: Parakeratosis was observed in the skin covers the ear of all members of both groups 2 and 3 (Fig. 3a).

Inflammatory cells infiltration: Skin epidermis of hydroquinone treated rabbits showed inflammatory cells infiltration mainly lymphocytes and eosinophils (Fig. 3b).
Severe congestion: Dermal blood vessels exhibited severe congestion. This alteration was observed mainly in the papillary layer.

Melanocytes alterations: Melanocytes were less predominant in the skin of the rabbits exposed to hydroquinon in the abdomen in comparison with the control ones. Also melanin granules of both basal and reticular layers were less concentrated in the melanocytes of hydroquinone treated animals in comparison to the control ones. Melanocytes in the drug exposed skin showed some shrinkage. 
Am. J. Pharm. \& Toxicol., 7 (1): 19-26, 2012

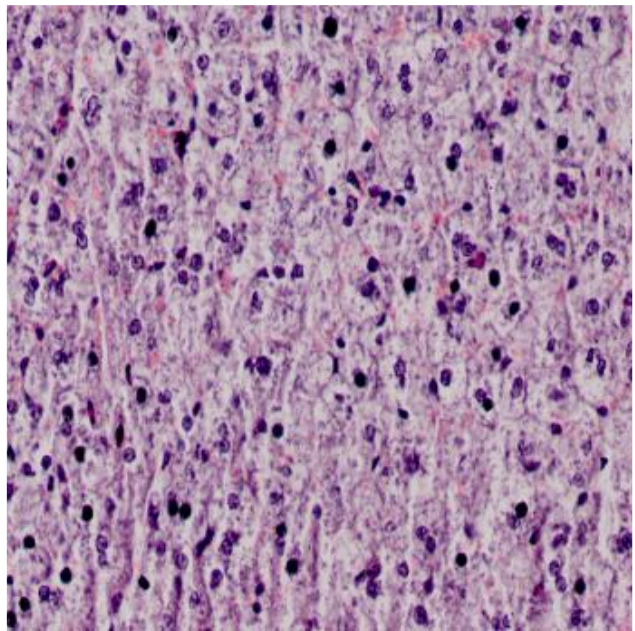

(a)

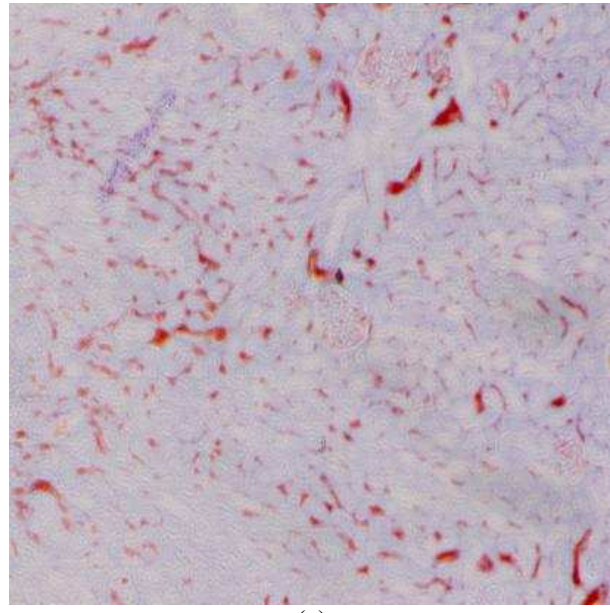

(c)

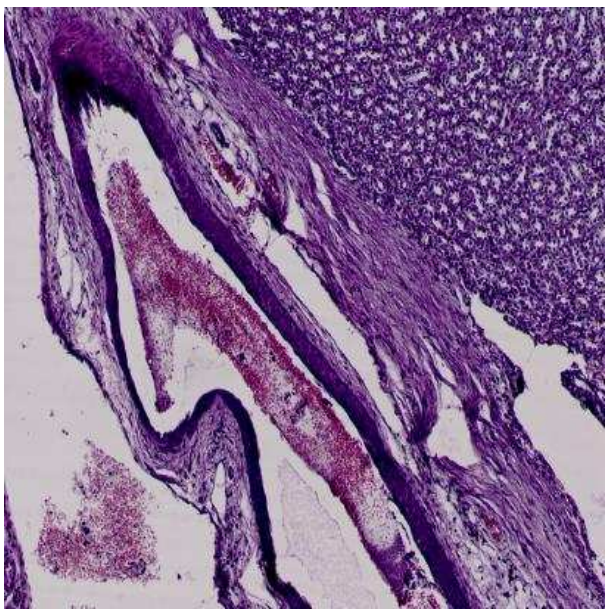

(e)

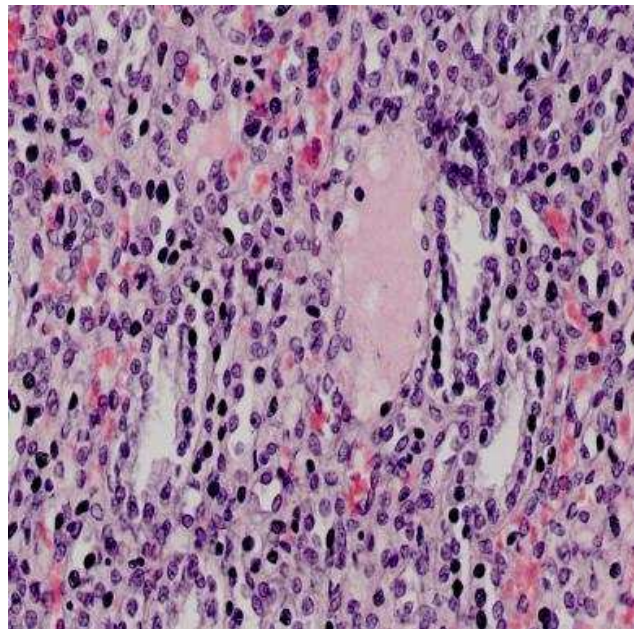

(b)

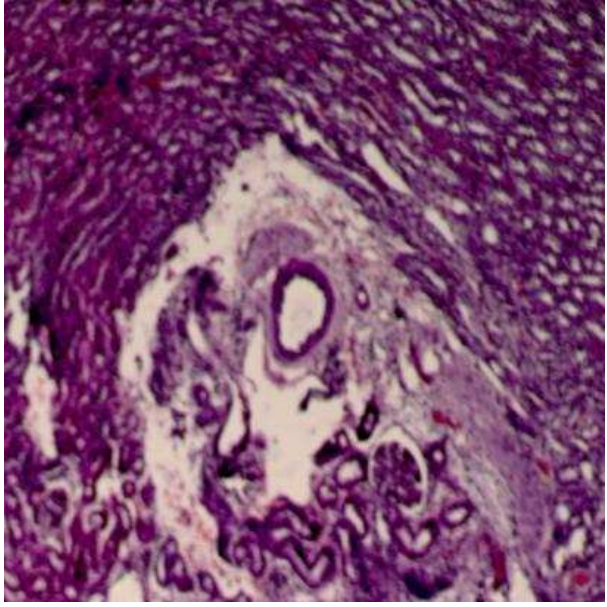

d)

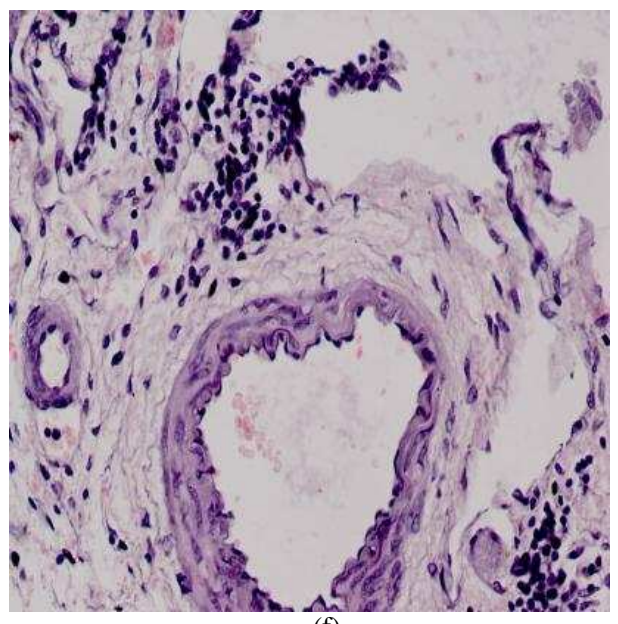

(f)

Fig. 2: Light micrograph section in the kidney of rabbit exposed to eldoquin (hydroquinone, 2\%, w/w) showing (a) Hydropic degeneration. H\&E stain; (b) Hyaline casts. H\&E stain; (c) Renal congestion; (d) Interstitial perivascular edema; (e) Perivascular fibrosis. Trichrome stain; (f) Perivascular lymphocytic aggregation. H\&E 


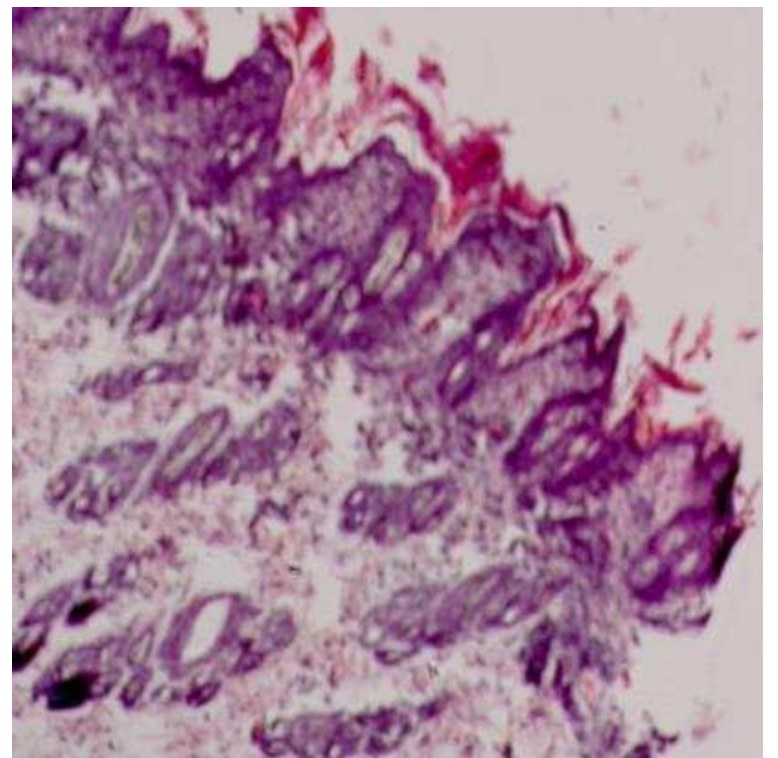

(a)

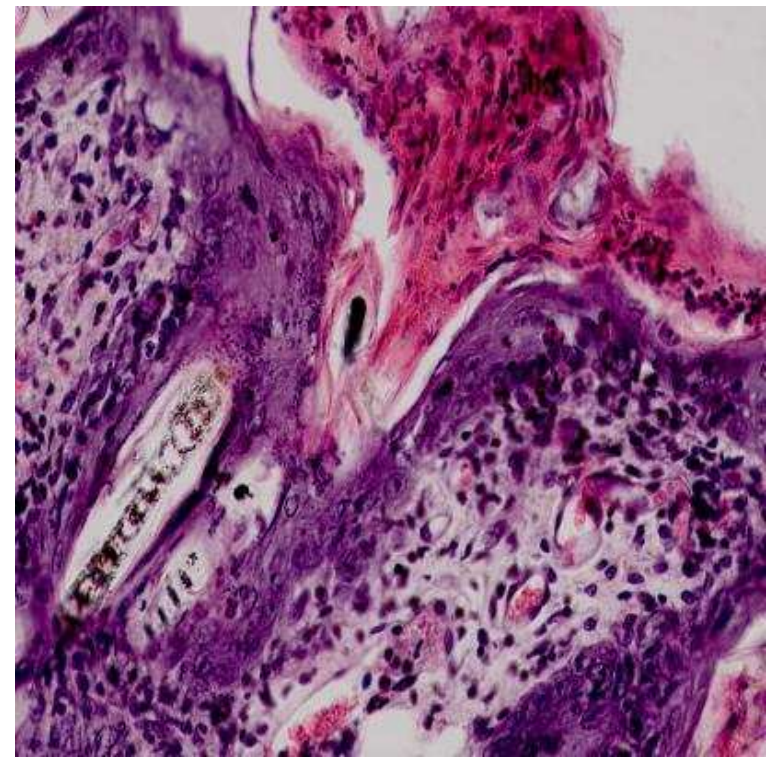

(b)

Fig. 3: Light micrograph section in the skin of rabbit exposed to eldoquin (hydroquinone, 2\%, w/w) showing (a) Hyperkeratosis H\&E stain; (b) Lymphocytic infiltration H\&E stain

The results of the present study indicate that hyroquinone caused several histological alterations in the liver mainly hydropic degeneration, bile duct hyperplasia and glycogen depletion. Some investigations indicated the liver to plays an important role in the detoxification of hydroquinone (Kooyers and Westerhof, 2006; Levitt, 2007). This drug is absorbed through the skin and metabolized to benzoquinone and multiple glutathione conjugates, that retain the ability to generate reactive oxygen species and detoxified subsequently via mercapturic acid formation (Barber et al., 1995; Wester et al., 1998; Poet et al., 2004; Lau et al., 2010).

The induced hydropic degeneration on the hepatocytes by hyroquinone might indicate toxic autolytic injury while the detected bile duct hyperplasia could be attributed to its irritation effect on the epithelium of the bile duct. The induced reduction in hepatocytes glycogen content by hydroquinone as seen in the results of the present study might be due to the effect of this drug on glucose absorption or on the enzymes involved in the process of glycogenesis or/and glycolysis. Hepatocytes of the periportal zones as seen in the present work were more affected than the perivenous hepatocytes. Accordingly, glycogenesis was more affected than glycolysis in which glucose is metabolized by the perivenous cells that contain higher levels of glucokinase and pyruvate. Hepatocytes in the area surrounding the terminal afferent are mainly gluconeogenic, while those ones surrounding the terminal efferent venule are mainly glycolytic and lipolytic and are involved in biotransformation as general detoxification mechanism (Al-Mansour et al., 2009). Other studies showed that murine hepatic adenomas were induced by hydroquinone exposure (Levitt, 2007).

The findings of the present investigation showed that hydroquinone might cause congestion in renal blood vessels together with other alterations in the renal interstitial tissue mainly perivascular edema, perivascular fibrosis and perivascular lymphocytic aggregation. These alterations could be resulted from injury of renal blood vessels endothelia induced by hydroquinone. More over, the induced hydropic degeneration together with the formation of hyaline casts in the lumen of some renal tubules of treated rabbits might indicate an insult of the kidney functions. Hydroquinone was reported to produce renal tubule adenomas and chronic progressive nephropathy (Hard et al, 1997; Levitt, 2007; Anderson et al., 2010).

The data of the present work showed that the cortex is more affected than medulla by hyroquinone treatment. This could be partly due to uneven distribution of the hyroquinone in the tissue of the kidney where about $90 \%$ of the total renal blood flow enters the cortex via the blood stream (Jarrar, 2003). This may indicate that more hyroquinone might reach the cortex via the blood stream than that would enter the medulla. Also, the findings showed that the insult by hydroquinone was more prominent in the proximal 
convoluted tubules than the distal ones. This could be due to the fact that the proximal tubules are primary sites of reabsorption and active transport leading to higher concentration of hyroquinone in the epithelial lining of these tubules. These alterations might be a result of hydrolic changes in the renal tissue and suggest that hyroquinone intoxication is an injurious stimulus that yields to a partial failure in the ion pump transport of tubules cells which in turn produce tubular degeneration of proximal tubules cells.

The presence of hyaline casts in the lumen of the damaged tubules by hydroquinone is an indication of glomerulnephritis and represents partial failure of tubular reabsorption. The kidney is rich in acid glycosidase and proteolytic enzymes located in lysosomes and other subcellular components (Kirschbaum et al., 1973). Oral administration of hydroquinone caused significant hyperplasia of the renal pelvic transitional epithelium and induced renal cortical casts (Kari et al., 1992). However, some studies did not record any significant change in the kidney after dermal administration of hydroquinone in rats which might indicate species specific toxicity of hydroquinone (David et al., 1998; O'Donoghue, 2006; Topping et al., 2007).

\section{CONCLUSION}

The findings of the present study indicate that dermal application of the hyroquinone caused hyperkeratosis and dermal congestion together with inflammatory cellular infiltration mainly lymphocytes and eosinophils. This may indicate an allergic effect of this drug or its metabolites that can cause dermatitis. Hydroquinone inhibits tyrosinase impairing partly the conversion of tyrosin to melanin via dopaquinone (Nordlund, 2007). This might explain the reduction of melanin granules in the melanocytes of hydroquinone treated rabbits in comparison to the control ones. Electron microscopic studies indicated that hydroquinone topical application could decrease the formation of melanosomes and caused melanocytes necrosis (Jimbow et al., 1974).

The findings of the present work indicate that alterations were more prominent in rabbits subjected to hydroquinone on both auricles on comparison to the members of other treated groups. This might indicate faster and more rapid distribution of the drug via the ear auricle than the abdominal skin. Further investigations are needed to elaborate the histological, histochemical and ultrastructural alterations induced by hydroquinone. In addition, it is recommended to study the effect of hydroquinone topical administration among patients with liver and kidney diseases and to test the safety of using hydroquinone among elderly people, where liver and kidney functions are significantly decreased (Klotz, 2009).

\section{REFERENCES}

Al-mansour, M.I., N.M. Al-Otabi, S.S. Alarifi, S.A. Ibrahim and B.M. Jarrar, 2009. Histological and Histochemical Alterations induced by lead in the liver of the quail Coturnix coturnix. Toxicol. Environ. Chem., 91: 1191-1203. DOI: 10.1080/02772240802590285

Anderson, F.A., W.F. Bergfeld, D.V. Belsito, R.A. Hill and C.D. Klassen et al., 2010. Final amended safety assessment of hydroquinone as used in cosmetics. Int. J. Toxicol., 29: 274S-287S. DOI: $10.1177 / 1091581810385957$

Bancroft, J.D. and A. Stevens, 1990. Theory and Practice of Histological Techniques. 3rd Edn., Churchill Livingstone, Edinburgh, ISBN: 0443035598, pp: 726.

Barber, E.D., T. Hill and D.B. Schum, 1995. The percutaneous absorption of Hydroquinone (HQ) through rat and human skin in vitro. Toxicol. Lett. 80: 167-172. DOI: 10.1016/0378-4274(95)03393-Y

Boatman, R.J., 1996. Differences in the nephrotoxicity of hydroquinone among Fischer 344 and SpragueDawley rats and $\mathrm{B} 6 \mathrm{C} 3 \mathrm{~F} 1$ mice. J. Toxicol. Environ. Health, 47: 159-172. DOI: 10.1080/009841096161861

Boyle, J. and C.T.C. Kennedy, 1985. Leukoderma from hydroquinone. Contact Dermatitis, 13: 287-288. DOI: 10.1111/j.1600-0536.1985.tb02578.x

Boyle, J. and C.T.C. Kennedy, 1986. Hydroquinone concentrations in skin lightening creams. Br. J. Dermatol., 114: 501-504. DOI: 10.1111/j.13652133.1986.tb02857.x

Carleton, H.M., R.A.B. Drury and E.A. Wallington, 1980. Carleton's Histological Technique. 5th Edn., Oxford University Press, New York, ISBN-10: 0192613103 , pp: 520.

Costa, A., T.A. Moises, T. Cordero, C.R.T. Alves and J. Marmirori, 2010. Association of emblica, licorice and belides as an alternative to hydroquinone in the clinical treatment of melasma. An. Bras. Dermatol., 85: 613-620. DOI: $10.1590 / \mathrm{S} 0365-$ 05962010000500003

David, R.M., J.C. English, L.C. Totman, C. Moyer and J.L. O'Donoghue, 1998. Lack of nephrotoxicity and renal cell proliferation following subchronic dermal application of a hydroquinone cream. Food Chem. Toxicol., 36: 609-616. DOI: 10.1016/S0278-6915(98)00014-3 
DeCaprio, A.P., 1999. The toxicology of hydroquinone - relevance to occupational and environmental exposure. Crit. Rev. Toxicol., 29: 283-330. DOI: 10.1080/10408449991349221

Devillers, J., P. Boule, P. Vasseur, P. Prevot and R. Steiman et al., 1990. Environmental and health risks of hydroquinone. Ecotoxicol. Environ. Saf., 19: 327-354. DOI: 10.1016/0147-6513(90)90035-4

Draelos, Z.D., 2007. Skin lightening preparations and the hydroquinone controversy. Dermatol. Ther., 20: 308-313. DOI: 10.1111/j.1529-8019.2007.00144.x

Hard, G.C., J. Whysner, J.C. English, E. Zang and G.M. Williams, 1997. Relationship of hydroquinoneassociated rat renal tumors with spontaneous chronic progressive nephropathy. Toxicol. Pathol., 25: 132-143. DOI: 10.1177/019262339702500202

Hazel, B.A., C. Baum and G.F. Kalf, 1996. Hydroquinone, a bioreactive metabolite of benzene, inhibits apoptosis in myeloblasts. Stem Cells, 14: 730-742. DOI: 10.1002/stem.140730

Jarrar, B.M., 2003. Histological and histochemical alterations in the kidney induced by lead. Ann. Saudi. Med., 23: 10-15. PMID: 17146214

Jimbow, K., H. Obata, M.A. Pathak and T.B. Fitzpatrick, 1974. Mechanism of depigmentation by hydroquinone. J. Invest. Dermat. 62: 436-449. DOI: 10.1111/1523-1747.ep12701679

Joseph, P., A.J.P. Klein-Szanto and A.K. Jaiswal, 1998. Hydroquinones cause specific mutations and lead to cellular transformation and in vivo tumorigenesis. Br. J. Cancer, 78: 312-320. DOI: 10.1038/bjc. 1998.492

Karamagi, C., E. Owino and E.T. Katabira, 2001. Hydroquinone neuropathy following use of skin bleaching cream: Case report. East Afr. Med. J., 78: 223-224. DOI: 10.4314/eamj.v78i4.9069

Kari, F.W., J. Bucher, S.L. Eustis, J.K. Haseman and J.E. Huff, 1992. Toxicity and carcinogenicity of hydroquinone in $\mathrm{F} 344 / \mathrm{N}$ rats and $\mathrm{B} 6 \mathrm{C} 3 \mathrm{~F}_{1}$ mice. Food. Chem. Toxicol., 30: 737-747. DOI: 10.1016/0278-6915(92)90075-V

Kirschbaum, B.B., P.W. Zoltick and H.B. Bosmann, 1973. Rat kidney acid hydrolase and glycoprotein: Glycosyltransferase activity in lead intoxication. Res. Comm. Path. Pharm., 5: 441-458. PMID: 4699477

Klotz, U., 2009. Pharmacokinetics and drug metabolism in the elderly. Drug. Metab. Rev., 41: 67-76. DOI: $10.1080 / 03602530902722679$

Kooyers, T.J. and W. Westerhof, 2006. Toxicology and health risks of hydroquinone in skin lightening formulations. J. Eur. Acad. Dermatol. Venereol., 20: 777-780. DOI: 10.1111/j.1468-3083.2005.01218.x
Lau, S.S., C.L. Kuhlman, S.B. Bratton and T.J. Monks, 2010. Role of hydroquinone-thiol conjugates in benzene-mediated toxicity. Chem. Biol. Interact., 184: 212-217. DOI: 10.1016/j.cbi.2009.12.016

Levitt, J., 2007. The safety of hydroquinone: A dermatologist's response to the 2006 Federal Register. J. Am. Acad. Dermatol., 57: 854-872. DOI: $10.1016 /$ j.jaad.2007.02.020

Makropoulos, V. and E.C. Alexopoulos, 2006. Case report: Hydroquinone and/or glutaraldehyde induced acute myeloid leukaemia? J. Occup. Med. Toxicol., 1: 19-19. PMID: 16872480

McGregor, D., 2007. Hydroquinone: An evaluation of the human risks from its carcinogenic and mutagenic properties. Crit. Rev. Toxicol., 37: 887914. DOI: 10.1080/10408440701638970

Murphy, S.J., R.E. Schroeder, A.M. Blacker, W.J. Krasavage and J.C. English, 1992. A study of developmental toxicity of hydroquinone in the rabbit. Fundam. Applied Toxicol., 19: 214-221. DOI: 10.1016/0272-0590(92)90154-A

Nordlund, J.J., 2007. Hydroquinone: Its value and safety. Commentary on the US FDA proposal to remove hydroquinone from the over-the-counter market. Expert Rev. Dermatol., 2: 283-287. DOI: 10.1586/17469872.2.3.283

O'Donoghue, J.L., 2006. Hydroquinone and its analogues in dermatology - a risk-benefit viewpoint. J. Cosmet. Dermatol., 5: 196-203. DOI: 10.1111/j.1473-2165.2006.00253.x

Pearse, A.G.E., 1985. Histochemistry: Theoretical and Applied. Analytical Technology. 4th Edn., Churchirll-Livingstone, Edinburgh, ISBN: 0443029970 , pp: 614.

Poet, T.S., H. Wu, J.C. English and R.A. Corley, 2004. Metabolic rate constants for hydroquinone in F344 rat and human liver isolated hepatocytes: Application to a PBPK model. Toxicol. Sci., 82: 925. DOI: $10.1093 /$ toxsci/kfh229

Ribeiro, A.L.T., A.L.B. Shimada, C.B. Hebad, T.F.D. Oliveira and A.P.D.M. Loureiro et al., 2011. In vivo hydroquinone exposure alters circulating neutrophil activities and impairs LPS-induced lung inflammation in mice. Toxicology, 288: 1-7. DOI: 10.1016/j.tox.2011.05.009

Taib, N.T., B.M. Jarrar and M.M. Mubarak, 2004. Ultrastructural alterations in renal tissues of rabbits induced by diclofenac sodium (Voltaren). Saudi. Med. J., 25: 1360-1365. PMID: 15494802

Topping, D.C., L.G. Bernard, J.L. O'Donoghue and J.C. English, 2007. Hydroquinone: Acute and subchronic toxicity studies with emphasis on neurobehavioral and nephrotoxic effects. Food Chem. Toxicol., 45: 70-78. DOI: 10.1016/j.fct.2006.07.019 
Wester, R.C., J. Melendres, X. Hui, R. Cox and S. Serranzana et al., 1998. Human in vivo and in vitro hydroquinone topical bioavailability, metabolism, and disposition. J. Toxicol. Environ. Health: Part A: Curr. Issues, 54: 301-317. DOI: $10.1080 / 009841098158863$

Westerhof, W. and T.J. Kooyers, 2005. Hydroquinone and its analogues in dermatology - a potential health risk. J. Cosm. Dermat., 4: 55-59. DOI: 10.1111/j.1473-2165.2005.40202.x
Zawar, V.P. and S.T. Mhaskar, 2004. Exogenous ochronosis following hydroquinone for melasma. J. Cosm. Dermat., 3: 234-236. DOI: 10.1111/j.14732130.2004.00089.x 